Research Article

\title{
Establishing a Method for Quantifying Spinal Curvature during Videofluoroscopic Swallow Studies: Applying the Modified Cobb Angle to Healthy Young and Older Adults
}

Ashwini M. Namasivayam-MacDonald ${ }^{1,{ }^{+},}{ }^{,}$, Luis F. Riquelme ${ }^{2,3,{ }^{\dagger}}$, Sonja M. Molfenter ${ }^{4,+}$

1. School of Rehabilitation Science, McMaster University, 1480 Main Street West, IAHS 420, Hamilton, Canada; E-Mail: namasia@mcmaster.ca

2. Speech-Language Pathology, New York Medical College, 40 Sunshine Cottage Road, Valhalla, NY, United States; E-Mail: luis riquelme@nymc.edu

3. Barrique Speech-Language Pathology, 3207 Avenue, \#308, Brooklyn, NY, United States; E-Mail: luisslp@me.com

4. Communicative Sciences and Disorders, New York University, 665 Broadway \#9, New York, United States; E-Mail: smm16@nyu.com

+ These authors contributed equally to this work.

* Correspondence: Ashwini M. Namasivayam-MacDonald; E-Mail: namasia@mcmaster.ca

Academic Editor: David G Smithard

Special Issue: Dysphagia in the Elderly

OBM Geriatrics

2020, volume 4, issue 3

doi:10.21926/obm.geriatr.2003129
Received: June 02, 2020

Accepted: July 27, 2020

Published: July 29, 2020

\begin{abstract}
The Cobb angle is traditionally used for quantifying the degree of spinal curvature through evaluation of the full spinal cord. When conducting measurements on videofluoroscopy swallowing studies (VFSS), the Cobb angle can measure degree of cervical vertebrae curvature, which may have implications for swallowing. Given that this measure may have utility in dysphagia research, the reliability of this measure taken from C2-C4 and establishing the presence of changes with age were the focus of the current, proof-ofprinciple study. VFSS from 19 healthy young adults and 39 healthy older adults were
\end{abstract}

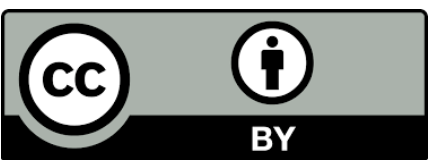

(C) 2020 by the author. This is an open access article distributed under the conditions of the Creative Commons by Attribution License, which permits unrestricted use, distribution, and reproduction in any medium or format, provided the original work is correctly cited. 
retrospectively analyzed. The C2-C4 Cobb angle was measured between cervical vertebrae two and four on frames of laryngeal vestibule closure (LVC) and post-swallow rest. Results revealed excellent levels of inter- and intra-rater reliability for frame of post-swallow rest (ICCS $=0.788$ and 0.793 ), and fair to good levels of agreement for frame of LVC (ICCS $=0.667$ and 0.621). Significant differences in the $\mathrm{C} 2-\mathrm{C} 4 \mathrm{Cobb}$ angle were found between the healthy young and old data $(p<0.01)$. Healthy younger adults had a mean angle of $5.8 \pm 9.0$ degrees at LVC and $7.7 \pm 4.5$ degrees at swallow rest, whereas healthy older adults had a mean angle of 12.5 \pm 9.0 degrees at LVC and 12.4 \pm 9.7 degrees at rest. Consistent with the existing spine literature, the curvature of cervical vertebrae appears to increase with age. With established reliability, we propose that the $\mathrm{C} 2-\mathrm{C} 4 \mathrm{Cobb}$ angle may be used to determine the degree of spinal curvature in a variety of patient populations in order to determine impacts on swallowing function.

\section{Keywords}

Deglutition; dysphagia; healthy adults; spine; assessment; videofluoroscopy

\section{Introduction}

Given the rapidly growing population of older adults, it is important to consider how natural and expected changes to the body - specifically to the head and neck - due to aging, might impact swallowing function. Research has previously established that with age we can expect changes to pharyngeal lumen size [1], tongue strength [2] and timing of the swallow [3], amongst other things. Something rarely considered when examining swallowing in older adults is their posture, which is influenced by the shape or curvature of the spine [4]. Interestingly, most previous research suggests that body position has little impact on swallowing $[5,6]$. However, one study by Su and colleagues quantified swallowing parameters in both an upright and supine position and determined that positioning had little impact on swallowing a thicker consistency, like pudding, but saw increases in temporal measures when thin liquids were swallowed in the supine position [7]. Anecdotally, clinicians encourage patients to sit upright as close to 90 degrees as possible in order to promote optimal swallowing function. They suggest that any sitting position less than 90 degrees may prevent the efficient passage of the bolus from the oral cavity into the esophagus [810]. The focus is on the sitting position and resulting posture, with little consideration of how the natural shape of the spine might influence swallowing function.

Studies analyzing anteriorly protruding cervical osteophytes, which are bony protrusions on the anterior surface of the lower cervical vertebrae, suggest that such changes to the spine impinge bolus passage into the cervical esophagus leading to increased pharyngeal residue and reduced upper esophageal sphincter opening [11-13], and/or altered sensation [14-16], as well as laryngeal penetration $[17,18]$. Other than this research, few studies outside of the traumatic spinal cord injury literature have examined the relationship between the changes to the spine and its impact on swallowing physiology and mechanics. Given that swallowing function post-spinal cord injury is dependent on the cause and type of injury [19], we cannot use such studies to make inferences about how non-injury related differences in spinal curvature might affect swallowing. Research 
specific to the cervical spine has suggested that kyphosis may result in pharyngeal phase deficits [20] and increased risk of aspiration [21]. Moreover, spinal-specific research has previously established that as one ages the degree of kyphosis (i.e. outward curvature of the spine causing "hunching" of the back [see Figure 1]) is expected to increase along the full spine [22-24]. Other research has postulated that neck posture can influence one's risk of aspiration [25-30]. For example, a few studies have suggested that neck flexion in the form of a chin-down posture improves laryngeal vestibule closure and epiglottic angle, resulting in reduced incidences of airway invasion [25, 31, 32]. Since kyphosis is expected with age and neck flexion may act as a protective mechanism during swallowing, one might assume that age-related changes in the spine might positively influence swallowing physiology.

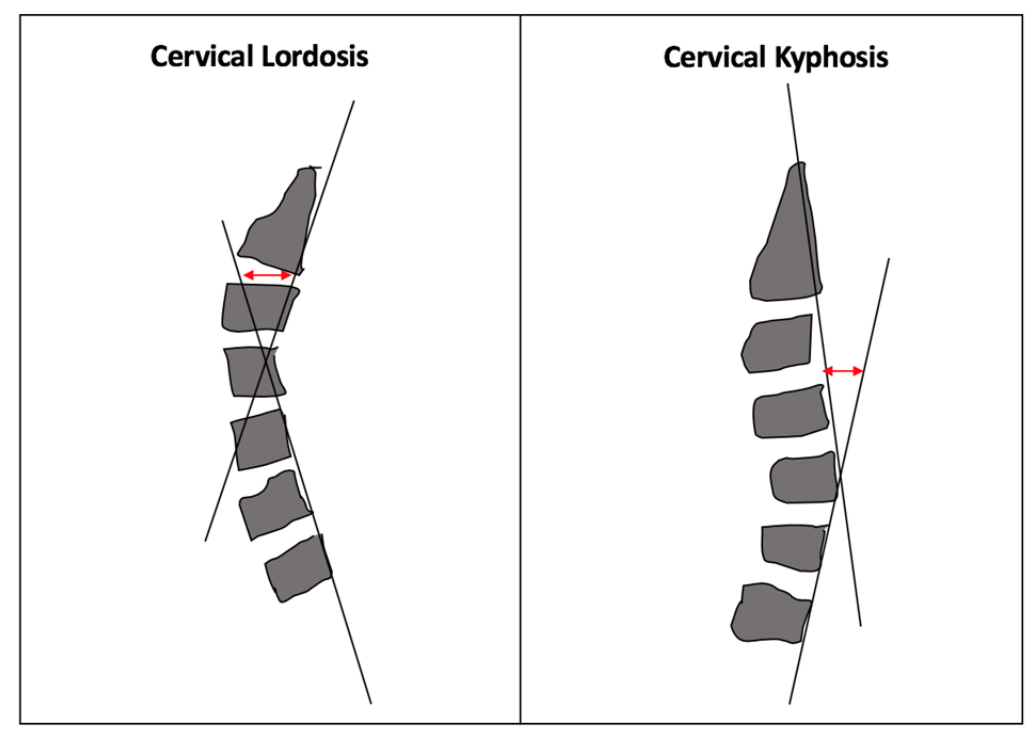

Figure 1 A lordotic spine (left) versus kyphotic cervical spine (right).

The spinal cord literature has provided some evidence of age-related changes to the cervical spine. A recent meta-analysis examined the existence and extent of cervical lordosis in asymptomatic individuals, and evaluated the relationship of this lordosis with age and gender [33]. Upon analyzing 21 studies, the authors found curvature was not significantly different between symptomatic and asymptomatic individuals, and age was not significantly associated with amount of lordotic cervical curvature. Interestingly, other studies have found that the angle of cervical lordosis tends to increase with age [34-37]. The parameter of interest for all of these studies was cervical lordosis - where the spine is curving posteriorly (see the image to the left of Figure 1). However, it is unknown whether kyphosis (anterior curvature or forward head posture) is seen specifically in the cervical vertebrae. These studies also all employed the C2-C7 (cervical vertebra two to cervical vertebra seven) Cobb angle to measure cervical curvature [38].

The Cobb angle has historically been used to quantify the degree of spinal curvature for patients with scoliosis through evaluation of the full spinal cord and has been established as reliable in this context [39]. This method defines angles between $20^{\circ}$ and $60^{\circ}$ as normal cervical curvature [20]. Typically, to measure the Cobb angle, a right angle is drawn between the top-most and bottom-most curved vertebrae, and measure the angle formed between the intersecting rays from each right angle (Figure 2). The $\mathrm{C} 2-\mathrm{C} 7 \mathrm{Cobb}$ method measures the curvature of the full 
cervical spine using radiographic images [39]. Videofluoroscopy swallowing studies (VFSS) are dynamic x-ray videos that are used to assess swallowing physiology and mechanics, with radiographic images taken depicting the region of the nasal cavity to the cervical esophagus. While a VFSS results in series of radiographic images, more often than not, all cervical vertebrae are not visible. More specifically, it is often difficult to clearly discern C5-C7. Given that C4 (cervical vertebra 4) is generally much more readily visible, we wanted to determine if the Cobb angle could be measured between $\mathrm{C} 2$ and $\mathrm{C} 4$, rather than between $\mathrm{C} 2$ and $\mathrm{C} 7$. In essence, the $\mathrm{C} 2-\mathrm{C} 4$ would act as a proxy measure, allowing a clinician to screen for cervical spine changes using VFSS data, rather than subjecting patients to a separate $x$-ray study of the spine. Interestingly, measures conducted between C2-C4 have become common in the dysphagia literature [40-44], likely due to the fact that they are clearly visible on VFSS, which is the gold standard method for evaluating swallowing. Since we do not currently have an established method for determining degree of cervical curvature on frames taken from VFSS, this was the primary purpose of the current, exploratory, proof-of-principle study. As a first step in this concept development, we aimed to establish if measurements taken from $\mathrm{C} 2$ to $\mathrm{C} 4$ were reliable. This would help to determine the potential of using the modified method as a proxy measure, instead of the traditional C2-C7 measurement. If the $\mathrm{C} 2-\mathrm{C} 4$ measurements were found to be reliable, we also aimed to determine if differences in cervical spine curvature measured were seen between healthy young and healthy old participants, consistent with previous spinal cord literature. Moreover, C2-C4 is highly correlated with participant height and thus frequently used in dysphagia research for scaling measures $[40,45]$. We hypothesized that a) we could reliably measure the Cobb angle between C2 and $\mathrm{C} 4$ and $\mathrm{b}$ ) that we would see a significant increase in the Cobb angle with age.

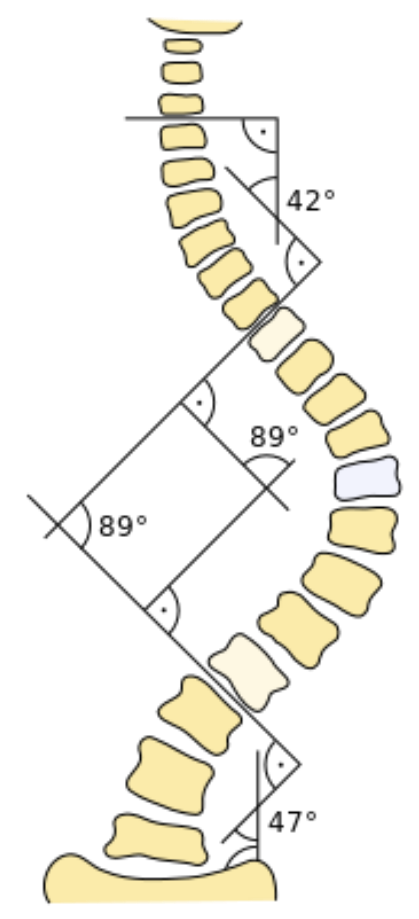

Figure 2 This image depicts the typical method for measuring the Cobb angle. This image by Skoliose-Info-Forum.de is licensed under the Creative Commons AttributionShare Alike 3.0 Unported license. 


\section{Materials and Methods}

\subsection{Data Collection}

The inclusion criteria for the database were that participants were either young adults between 18 and 45 years of age and healthy, or older healthy adults above 65 years of age. The database consisted of videos used for the sole purpose of research. Exclusion criteria for all participant in the database included a history of dysphagia, neurological disease/insult, significant head and neck surgery (other than routine dental surgery, tonsillectomy, or adenoidectomy), major spinal deformities, chemoradiation to the head and neck, and/or possible pregnancy. Ultimately, VFSS were included from 19 healthy young adults (10 males; mean age: 32; range: 22-45) and 39 healthy older adults (18 males; mean age: 77; range: 65-95). VFSS were excluded if image quality and/or positioning of the participant prevented the spine from being viewed clearly $(n=6,1$ younger adult and 5 older adults). All studies were conducted using a Kay Pentax Digital Swallow Workstation recording system, with the fluoroscope in lateral view at 30 pulses per second and were captured and recorded at 30 frames per second.

Data for this study were extracted from a retrospective research archive of VFSS. The original studies were approved by the research ethics boards at Toronto Rehabilitation Institute and New York University, and written consent was obtained from each participant prior to study participation.

\subsection{Data Processing}

Data extraction for this study was restricted to clips of the first, single sip of thin liquid for each participant (continuous cup and straw drinking were excluded). Using standard desktop computers with i7 processors and labs with dim lighting so that the $x$-ray images were clearly visible, the VFSS were first spliced into bolus-level clips using Corel Video Studio Pro. They were then assigned an alphanumeric code. Raters were a speech-language pathologist and a speech-language pathology graduate student who had 4 years and 1 year, respectively, of previous experience performing frame-by-frame analyses of VFSS, including identifying swallowing physiology, events and kinematics [41]. For the purposes of this study, raters underwent further training on how to measure the Cobb angle. Given the potential for spine angle differences during swallowing and at rest, the raters were asked to identify two frames per participant. First, they identified the frame of maximum laryngeal vestibule closure (LVC), defined as the first frame where there was maximum approximation of the arytenoids to the laryngeal surface of the epiglottis. They also identified the frame of post-swallow rest, defined as the first frame showing the pyriform sinuses at the lowest position, relative to the spine, prior to any hyoid burst or laryngeal elevation for a subsequent subswallow. These two frames were chosen with the intent of capturing the spine angle during the height of the swallow (LVC; i.e. during swallowing) and capturing the spine angle at rest where it would be unlikely for the posture to be influenced by swallowing. Post-swallow rest, rather than pre-swallow bolus hold, was chosen as a frame of rest given that data were extracted retrospectively and not all participants performed bolus holds during their VFSS. Further, the ASPEKT (Analysis of Swallowing Physiology: Events, Kinematics and Timing) method [41] is regularly employed in the lab, and this method requires several measures to be taken at the frame 
of LVC and at the frame of post-swallow rest. Given the high reliability of identifying these frames, we felt it best to choose these frames for the current study.

\subsection{Videofluoroscopy Rating}

Once frames of LVC and post-swallow rest were identified and agreed upon, the degree of cervical spine curvature was measured using the Cobb angle. For the current study, the Cobb angle was measured between $\mathrm{C} 2$ and $\mathrm{C} 4$ in lateral-view VFSS using the angle tool in ImageJ (https://imagej.nih.gov/nih-image/) on the pre-established frames of LVC and post-swallow rest for thin liquid swallows (Figure 3). These vertebrae were chosen because they tend to be consistently and most easily visible on a VFSS. Further, C7 was not visible in any of the VFSS in our database, likely due to the fact that the shoulder generally obstructs the view of the inferior cervical spine when patients are positioned in the lateral view. C2-C4 appeared far enough apart to obtain a measure of curvature. Raters used the ROI Manager within ImageJ to allow each angle drawn to remain on the screen while subsequent angles were drawn, and measurements were taken. Twenty percent of the measures were taken in duplicate in order to calculate inter- and intra-rater reliability.

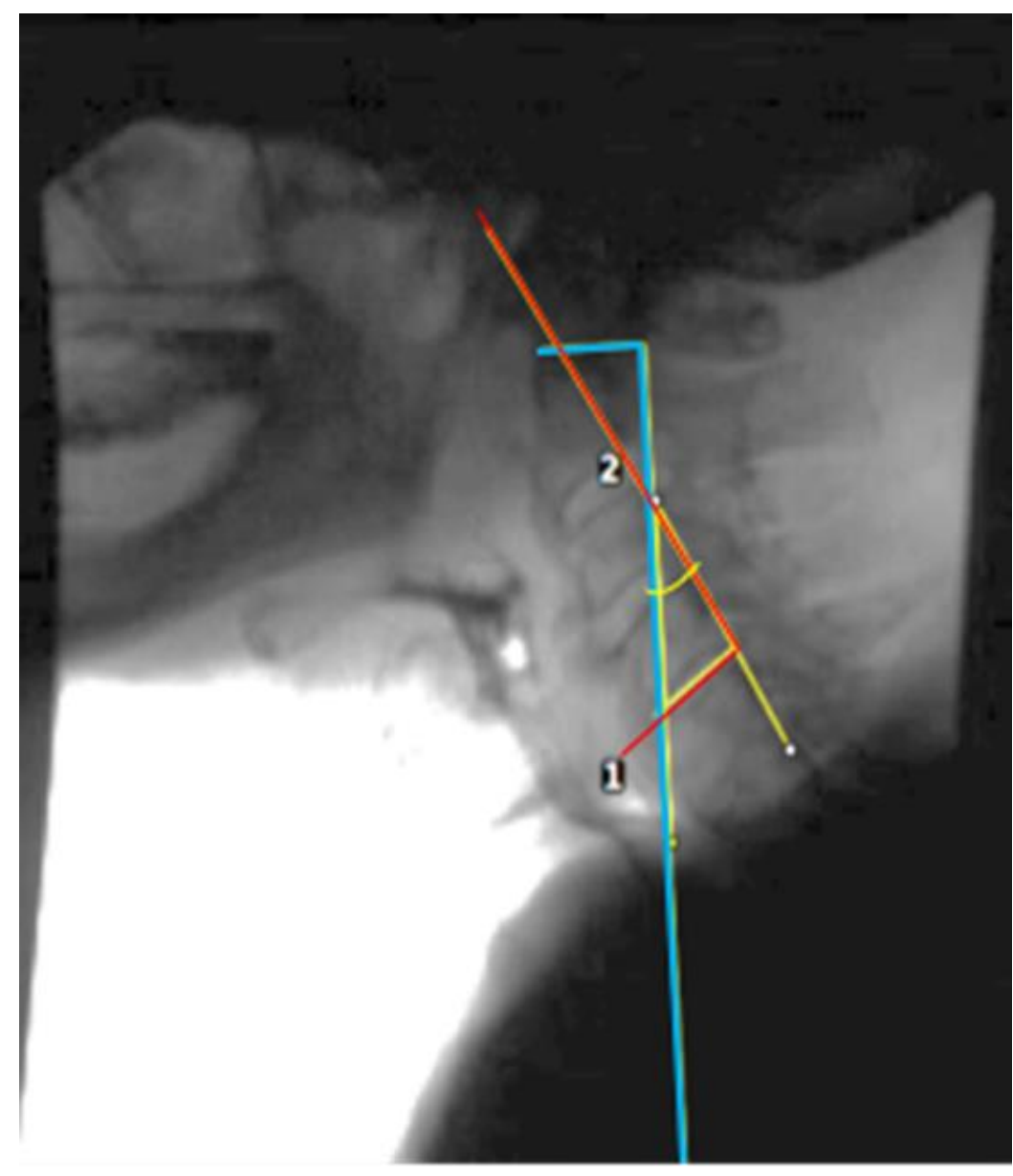

Figure 3 Example of two $90^{\circ}$ angles around superior portion of C2 (angle 2, in blue) and the inferior portion of $\mathrm{C} 4$ (angle 1 , in red) intersect to measure the Cobb angle (in yellow). 


\subsection{Data Analysis}

All statistical analyses were conducted in IBM SPSS version 25. First, all reliability measures were computed using two-way mixed intraclass correlation coefficients (ICCS) for consistency. A priori determined cut-offs for acceptable reliability were established. Intra- and inter-rater reliability assess agreement of the same rater with themselves (intra) or with a second rater (inter). Intra- and inter-rater reliability ICCs of 0.75 and above are considered to have 'excellent' reliability [46]. Next, descriptive statistics were calculated to describe mean cervical curvature for each participant group at both frame of LVC and frame of post-swallow rest. Independent sample ttests were used to compare degree of curvature at each time point between healthy young and healthy old. Two-tailed $p$-values $p<.05$ were considered statistically significant. Effect sizes for significant pairwise comparisons were calculated using Cohen's $d$. Cohen's $d$ can be interpreted as showing a small effect size for values of $<0.5$, medium effect size for values of $0.5-0.8$, and large effect size for values of $>0.8$ [47].

\section{Results}

Results revealed excellent levels of agreement within and across raters for degree of C2-C4 curvature on the frame of post-swallow rest $(\mathrm{ICC}=0.793(95 \% \mathrm{Cl}[-0.032,0.959])$ and $0.788(95 \% \mathrm{Cl}$ $[0.060,0.952])$, respectively). There were fair to good levels of agreement within and across raters for frame of LVC (ICC $=0.621(95 \% \mathrm{Cl}[-0.893,0.924])$ and $0.667(95 \% \mathrm{Cl}[-0.476,0.925])$, respectively).

Descriptive statistics for the parameters of interest (modified Cobb's angle measured at LVC and post-swallow rest) are displayed in Table 1. Significant differences in the C2-C4 Cobb angle were found between healthy young and healthy old at post-swallow rest $(t(55)=2.035, p=0.003$; Cohen's $d=0.633$ [medium]). Significant differences in the C2-C4 Cobb angle were also found between the healthy young and healthy old data at frame of $\operatorname{LVC}(\mathrm{t}(56)=3.140, \mathrm{p}=0.001$; Cohen's $d=0.97$ [large]). No significant differences were found between C2-C4 Cobb angle measured from frame of LVC compared to frame of post-swallow rest across collapsed age groups $(t(81)=-0.809$, $p=0.421)$.

Table 1 Descriptive statistics for the modified Cobb angle measured across samples at two different timepoints: laryngeal vestibule closure (LVC) and post-swallow rest.

\begin{tabular}{|cccc|}
\hline Measure & $\begin{array}{c}\text { Healthy young } \\
(\mathbf{n}=19)\end{array}$ & $\begin{array}{c}\text { Healthy old } \\
(\mathbf{n}=\mathbf{3 9})\end{array}$ & P-value \\
\hline $\begin{array}{c}\text { C2-C4 angle at } \\
\text { frame of LVC }\end{array}$ & $5.8 \pm 9.0^{\circ}$ & $12.5 \pm 9.0^{\circ}$ & $0.001^{*}$ \\
$\begin{array}{c}\text { (mean } \pm \text { standard deviation) } \\
\text { C2-C4 angle at }\end{array}$ & & $12.4 \pm 9.7^{\circ}$ & $0.003^{*}$ \\
$\begin{array}{c}7.7 \pm 4.5^{\circ} \\
\text { frame of post-swallow rest } \\
\text { (mean } \pm \text { standard deviation) }\end{array}$ & & & \\
\hline
\end{tabular}

*Denotes a statistically significant $p$-value, per the results of $t$-test. 


\section{Discussion}

The primary objectives of this proof-of-principle study were to establish a reliable method for measuring cervical spine curvature on VFSS and determine if differences existed in cervical spine curvature between healthy young and healthy old samples. Our findings revealed that the C2-C4 Cobb angle may be a reliable method to measure cervical spine curvature, particularly when measured on frame of post-swallow rest. In addition, we found significant differences in curvature between younger and older healthy participants. More specifically, consistent with the existing spinal cord literature, cervical vertebrae two to four appear to increase in curvature with age [2224, 35-38].

It is interesting to note that reliability is more clearly established on frame of post-swallow rest compared to frame of LVC. The reduced reliability on frame of LVC could be due to the movement inherent within the pharyngeal stage of the swallow, causing artifact within that frame so that the borders of the cervical vertebrae were not completely clear to raters. The overall quality of the videos may have also played a role in making the necessary ratings. Given that no significant differences were found between the C2-C4 Cobb angle at frame of LVC compared to frame of swallow rest, we recommend that future use of the $\mathrm{C} 2-\mathrm{C} 4 \mathrm{Cobb}$ angle in this context be performed on frame of post-swallow rest over the frame of LVC. It may be warranted to explore other frames of interest in future research.

This non-invasive method of measuring cervical spinal cord curvature has potential use for future dysphagia research. With established reliability, the C2-C4 Cobb angle can be used to determine the degree of spinal curvature in a variety of populations and potentially explore its impact on swallow biomechanics. In order to further establish reliability, it is important that this method is validated by comparing the results to the standard application of the Cobb angle from C2-C7 and by evaluating the efficacy of this measurement in patients with documented cervical spine changes. Once confirmed to be reliable and valid, the modified method might specifically be useful in situations where we expect damage to or deterioration of the cervical spine, without having to request a separate $\mathrm{x}$-ray to take measurements on the full cervical spine using the $\mathrm{C2}-\mathrm{C} 7$ Cobb method. Moreover, the measurements can be incorporated into statistical analyses to control for variation attributed to spinal curvature during swallowing. They might also be used as a method of monitoring changes in spinal curvature within older adults along the course of a disease trajectory. Some might also choose to use measurements of $\mathrm{C} 2-\mathrm{C} 4$ spine curvature to help explain changes in the swallow that cannot be attributed to timing, coordination or basic kinematics. This proxy measure might eventually be useful for clinicians who are concerned about neck posture and would like to track changes in cervical spine curvature via clinical VFSS performed over time.

When considering that significant differences in $\mathrm{C} 2-\mathrm{C} 4$ curvature were found between the young and older participants, one might question the reason for these changes across the lifespan. Recent research by Brates et al. [40] suggests that changes to spinal morphology result in scaled hyoid movements that are inflated in older individuals because of a reduction in $\mathrm{C} 2$ to $\mathrm{C} 4$ length, due to cervical disc degeneration present in most older adults [48]. This degeneration results in a loss of intervertebral height. It is possible that this difference in C2 to C4 length may be impacting the Cobb angle, or an increase in kyphosis is contributing to a shorter cervical spine height. Future work will need to consider these age-related changes to the spine and clarify how kyphosis and 
disc degeneration are related. In order to monitor these changes over time, we will also need to establish test-retest reliability.

Given that the purpose of the current, exploratory study was to establish proof-of-principle, several limitations require acknowledgement. Firstly, the method itself has some limitations. Since it is measuring spine curvature between a relatively short distance ( 3 vertebrae), the resulting measurements may not account for disc protrusion, osteophytes, or other factors influencing spine curvature that occur below $\mathrm{C} 4$. However, given that the region between $\mathrm{C} 2$ and $\mathrm{C} 4$ is where many important physiologic swallowing functions take place, we predict that curvature in this area is significant for swallowing physiology and is therefore useful to measure. Another limitation is that the data analyzed for this study was restricted to a retrospective sample of young and older healthy adults, who did not have any documented cervical spine changes. A further limitation is that given that the data were extracted retrospectively from a research archive, it is possible that differences in head posture, patient positioning and/or instructions across participants may have impacted the findings. We were also unable to compare our C2-C4 Cobb angle measurements to measurements taken from $\mathrm{C2}-\mathrm{C7}$, given that C7 was not visible on the large majority of VFSS. Future studies should consider validation against $x$-ray where more of the spine is visible and/or MRI. Moving forward, it will be critical to perform a prospective study, where many of these factors can be controlled. Future studies should also consider minimizing random error by taking a minimum of three measurements and averaging the results.

\section{Conclusions}

The Cobb angle is a method of measuring spine curvature on x-rays and is a relatively simple measurement to conduct. This proof-of-principle research establishes that the Cobb angle measurements between cervical vertebrae two and four, derived from VFSS using ImageJ on the frame of post-swallow rest, have satisfactory inter-rater and intra-rater reliability. Moreover, we have confirmed changes in cervical spine curvature with age using this modified Cobb angle method, thus suggesting that the measure may be an option for monitoring cervical spine curvature via VFSS. Future work should focus on recruiting a prospective sample that includes patients with documented cervical spine changes that are age-matched with healthy participants. It would also be interesting to determine if the trend of increased $\mathrm{C2}-\mathrm{C} 4$ spine curvature continues with old, old adults (i.e. those who are $80+$ years of age). Given that cervical spine curvature may be influenced by posture and thoracic spine curvature, future work should compare these parameters to measurements taken using a cervical range of motion device, used to evaluate the range of motion of the cervical spine. Validation of this method within a prospective study using the C2-C7 Cobb method will also be an important next step towards using this adapted method in research and clinical practice. Lastly, future studies should determine if and at what point cervical kyphosis influences swallowing physiology. The directionality of the curvature must also be confirmed, as well as the implications of lordotic and kyphotic cervical spines on swallowing function in different populations and in various swallowing postures.

\section{Acknowledgments}

The authors would like to thank Danielle Brates and Alexandra Chill for their assistance with data collection and analysis. 


\section{Author Contributions}

ANM: conception, experiment design, data analysis, drafted, and revised manuscript; LFR: conception, experiment design, and revised manuscript; SMM: conception, experiment design, data collection, data analysis, and revised manuscript.

\section{Funding}

Data collection of the older healthy controls was supported by R21DC015067-01 (awarded to Molfenter).

\section{Competing Interests}

The authors have declared that no competing interests exist.

\section{References}

1. Molfenter SM, Lenell C, Lazarus CL. Volumetric changes to the pharynx in healthy aging: consequence for pharyngeal swallow mechanics and function. Dysphagia. 2019 ;34: 129-137.

2. Fei T, Polacco RC, Hori SE, Molfenter SM, Peladeau-Pigeon M, Tsang C, et al. Age-related differences in tongue-palate pressures for strength and swallowing tasks. Dysphagia. 2013; 28: 575-581.

3. Namasivayam-MacDonald AM, Barbon CEA, Steele CM. A review of swallow timing in the elderly. Physiol Behav. 2018; 184: 12-26.

4. Raine S, Twomey LT. Head and shoulder posture variations in 160 asymptomatic women and men. Arch Phys Med Rehabil. 1997; 78: 1215-1223.

5. Barkmeier JM, Bielamowicz S, Takeda N, Ludlow CL. Laryngeal activity during upright vs. supine swallowing. J Appl Physiol (1985). 2002; 93: 740-745.

6. Dejaeger E, Pelemans W, Ponette E, Vantrappen G. Effect of body position on deglutition. Dig Dis Sci. 1994; 39: 762-765.

7. Su HK, Khorsandi A, Silberzweig J, Kobren AJ, Urken ML, Amin MR, et al. Temporal and physiologic measurements of deglutition in the upright and supine position with videofluoroscopy (VFS) in healthy subjects. Dysphagia. 2015; 30: 438-444.

8. Palmer JB, Kuhlemeier KV, Tippett DC, Lynch C. A protocol for the videofluorographic swallowing study. Dysphagia. 1993; 8: 209-214.

9. Alghadir AH, Zafar H, Al-Eisa ES, Iqbal ZA. Effect of posture on swallowing. Afr Health Sci. 2017; 17: 133-137.

10. Sutthiprapaporn $P$, Tanimoto $K$, Ohtsuka $M$, Nagasaki $T$, lida $Y$, Katsumata A. Positional changes of oropharyngeal structures due to gravity in the upright and supine positions. Dentomaxillofac Radiol. 2008; 37: 130-135.

11. Granville $\amalg$, Musson N, Altman R, Silverman M. Anterior cervical osteophytes as a cause of pharyngeal stage dysphagia. J Am Geriatr Soc. 1998; 46: 1003-1007.

12. Stuart D. Dysphagia due to cervical osteophytes. Int Orthop. 1989; 13(2): 95-99.

13. Yin $T$, Jardine $M$, Miles A, Allen J. What is a normal pharynx? A videofluoroscopic study of anatomy in older adults. Eur Arch Otorhinolaryngol. 2018; 275: 2317-2323. 
14. McCulloch TM, Jaffe D. Head and neck disorders affecting swallowing. GI Motility online. 2006.

15. Kim MJ, Chung TS, Lee JT, Yoo HS, Suh JH, Chang TY, et al. Radiologic evaluation of the globus symptom using videotape recorder. J Korean Radiol Soc. 1988; 24: 381-389.

16. Egerter AC, Kim ES, Lee DJ, Liu JJ, Cadena G, Panchal RR, et al. Dysphagia secondary to anterior osteophytes of the cervical spine. Global Spine J. 2015; 5: e78-e83.

17. Seidler TO, Alvarez JP, Wonneberger K, Hacki T. Dysphagia caused by ventral osteophytes of the cervical spine: clinical and radiographic findings. Eur Arch Otorhinolaryngol. 2009; 266: 285-291.

18. Frempong-Boadu A, Houten JK, Osborn B, Opulencia J, Kells L, Guida DD, et al. Swallowing and speech dysfunction in patients undergoing anterior cervical discectomy and fusion: $A$ prospective, objective preoperative and postoperative assessment. J Spinal Disord Tech. 2002; 15: 362-368.

19. Valenzano TJ, Waito AA, Steele CM. Characterizing dysphagia and swallowing intervention in the traumatic spinal injury population. Dysphagia. 2016; 31: 598-609.

20. Papadopoulou S, Exarchakos G, Beris A, Ploumis A. Dysphagia associated with cervical spine and postural disorders. Dysphagia. 2013; 28: 469-480.

21. Kim SK, Mo SJ, Moon WS, Jun PS, Kim CR. Effects of cervical kyphosis on recovery from dysphagia after stroke. Ann Rehabil Med. 2016; 40: 816-825.

22. Hinman MR. Comparison of thoracic kyphosis and postural stiffness in younger and older women. Spine J. 2004; 4: 413-417.

23. Katzman WB, Wanek L, Shepherd JA, Sellmeyer DE. Age-related hyperkyphosis: Its causes, consequences, and management. J Orthop Sports Phys Ther. 2010; 40: 352-360.

24. Fon G, Pitt M, Thies A. Thoracic kyphosis: Range in normal subjects. Am J Roentgenol. 1980; 134: 979-983.

25. Shanahan TK, Logemann JA, Rademaker AW, Pauloski BR, Kahrilas PJ. Chin-down posture effect on aspiration in dysphagic patients. Arch Phys Med Rehabil. 1993; 74: 736-739.

26. Rasley A, Logemann JA, Kahrilas PJ, Rademaker AW, Pauloski BR, Dodds WJ. Prevention of barium aspiration during videofluoroscopic swallowing studies: Value of change in posture. Am J Roentgenol. 1993; 160: 1005-1009.

27. Channer KS, Virjee J. Effect of posture and drink volume on the swallowing of capsules. $\mathrm{Br}$ Med J. 1982; 285: 1702.

28. McFarland DH, Lund JP, Gagner M. Effects of posture on the coordination of respiration and swallowing. J Neurophysiol. 1994; 72: 2431-2437.

29. Tsukada T, Taniguchi H, Ootaki S, Yamada Y, Inoue M. Effects of food texture and head posture on oropharyngeal swallowing. J Appl Physiol. 2009; 106: 1848-1857.

30. Logemann JA, Rademaker AW, Pauloski BR, Kahrilas PJ. Effects of postural change on aspiration in head and neck surgical patients. Otolaryngol Head Neck Surg. 1994; 110: 222227.

31. Ekberg O. Closure of the laryngeal vestibule during deglutition. Acta Otolaryngol. 1982; 93: 123-129.

32. Leigh JH, Cho K, Barcenas CL, Paik NJ. Dysphagia Aggravated by cervical hyperlordosis. Am J Phys Med Rehabil. 2011; 90: 704.

33. Guo GM, Li J, Diao QX, Zhu TH, Song ZX, Guo YY, et al. Cervical lordosis in asymptomatic individuals: A meta-analysis. J Orthop Surg Res. 2018; 13: 147. 
34. Chen Y, Luo J, Pan Z, Yu L, Pang L, Zhong J, et al. The change of cervical spine alignment along with aging in asymptomatic population: A preliminary analysis. Eur Spine J. 2017; 26: 23632371.

35. Attiah M, Gaonkar B, Alkhalid Y, Villaroman D, Medina R, Ahn C, et al. Natural history of the aging spine: $A$ cross-sectional analysis of spinopelvic parameters in the asymptomatic population. J Neurosurg Spine. 2019; 32: 63-68.

36. Kim HJ, Lenke LG, Oshima Y, Chuntarapas T, Mesfin A, Hershman S, et al. Cervical lordosis actually increases with aging and progressive degeneration in spinal deformity patients. Spine Deform. 2014; 2: 410-414.

37. Tang R, Ye IB, Cheung ZB, Kim JS, Cho SK-W. Age-related Changes in Cervical Sagittal Alignment: A Radiographic Analysis. Spine. 2019; 44: E1144-E1150.

38. Harrison DE, Harrison DD, Cailliet R, Troyanovich SJ, Janik TJ, Holland B. Cobb method or Harrison posterior tangent method: Which to choose for lateral cervical radiographic analysis. Spine. 2000; 25: 2072-2078.

39. Tanure MC, Pinheiro AP, Oliveira AS. Reliability assessment of Cobb angle measurements using manual and digital methods. Spine J. 2010; 10: 769-774.

40. Brates D, Steele C, Molfenter SM. Measuring hyoid excursion across the life span: Anatomical scaling to control for variation. J Speech Lang Hear Res. 2020; 63: 125-134.

41. Steele CM, Peladeau-Pigeon M, Barbon CAE, Guida BT, Namasivayam-MacDonald AM, Nascimento WV, et al. Reference values for healthy swallowing across the range from thin to extremely thick liquids. J Speech Lang Hear Res. 2019; 62: 1338-1363.

42. Molfenter SM, Steele CM. The relationship between residue and aspiration on the subsequent swallow: an application of the normalized residue ratio scale. Dysphagia. 2013; 28: 494-500.

43. Pearson WG, Molfenter SM, Smith ZM, Steele CM. Image-based measurement of postswallow residue: The normalized residue ratio scale. Dysphagia. 2013; 28: 167-177.

44. Stokely SL, Peladeau-Pigeon M, Leigh C, Molfenter SM, Steele CM. The relationship between pharyngeal constriction and post-swallow residue. Dysphagia. 2015; 30: 349-356.

45. Molfenter SM, Steele CM. Use of an anatomical scalar to control for sex-based size differences in measures of hyoid excursion during swallowing. J Speech Lang Hear Res. 2014; 57: 768-778.

46. Fleiss JL. Design and analysis of clinical experiments. John Wiley \& Sons; 2011.

47. Kotrlik J, Williams $H$. The incorporation of effect size in information technology, learning, information technology, learning, and performance research and performance research. Inform Technol Learn Perform J. 2003; 21: 1.

48. Friedenberg ZB, Edeiken J, Spencer HN, Tolentino SC. Degenerative changes in the cervical spine. JBJS. 1959; 41: 61-102. 


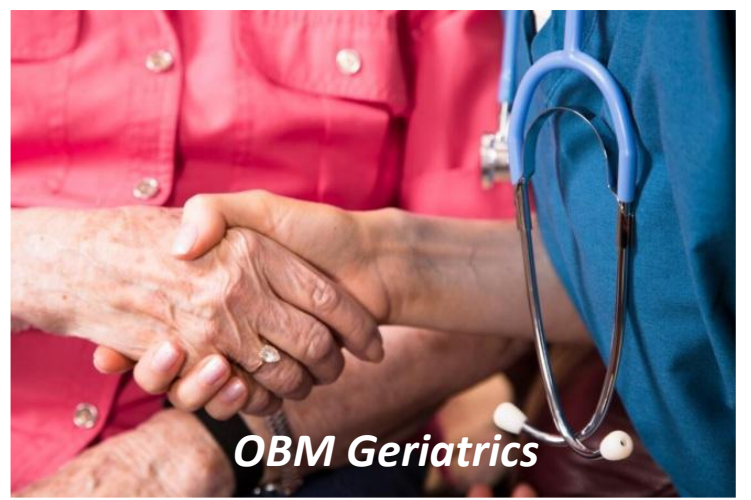

Enjoy OBM Geriatrics by:

1. Submitting a manuscript

2. Joining in volunteer reviewer bank

3. Joining Editorial Board

4. Guest editing a special issue

For more details, please visit:

http://www.lidsen.com/journals/geriatrics 